\title{
Belphégor
}

\section{Emilio Ghione, Za la Mort. Romanzo. A cura di Denis}

Lotti

Cuneo: Nerosubianco Edizioni, 2012. 235 p. ISBN 9-788898-007028

\section{Vittorio Frigerio}

\section{(2) OpenEdition}

\section{Journals}

Edizione digitale

URL: http://journals.openedition.org/belphegor/234

DOI: 10.4000/belphegor.234

ISSN: 1499-7185

Editore

LPCM

Notizia bibliografica digitale

Vittorio Frigerio, «Emilio Ghione, Za la Mort. Romanzo. A cura di Denis Lotti », Belphégor [En ligne], 11-1 | 2013, mis en ligne le 22 juin 2013, consulté le 22 septembre 2020. URL : http://

journals.openedition.org/belphegor/234; DOI : https://doi.org/10.4000/belphegor.234

Questo documento è stato generato automaticamente il 22 settembre 2020.

\section{cc) $(1)$}

Belphégor est mis à disposition selon les termes de la Licence Creative Commons Attribution - Pas d'Utilisation Commerciale - Pas de Modification 4.0 International. 


\section{Emilio Ghione, Za la Mort. Romanzo.} A cura di Denis Lotti

Cuneo: Nerosubianco Edizioni, 2012. 235 p. ISBN 9-788898-007028

Vittorio Frigerio

NOTIZIA

Emilio Ghione. Za la Mort. Romanzo. A cura di Denis Lotti. Cuneo: Nerosubianco Edizioni, 2012. 235 p. ISBN 9-788898-007028 
1 La gente non legge più. Allora forse tanto vale ripubblicare un libro dei tempi quando la gente leggeva, e le si diceva oltretutto che questa roba non avrebbe dovuto leggerla, pur sapendo che probabilmente, al giorno d'oggi, non lo leggerà nessuno. $\mathrm{O}$ perlomeno non lo leggerà nessuno di quelli che comunque non leggerebbero neanche altro, né nessuno di quelli che si immaginano ancora essere coloro che determinano cosa si debba e cosa non si debba leggere sotto l'etichetta di letteratura. Il che lascia gli altri, e speriamo che siano numerosi, per ricompensare l'audace editore e, semplicemente, perchè un po' di sano piacere si vorrebbe che tutti possano gioirne.

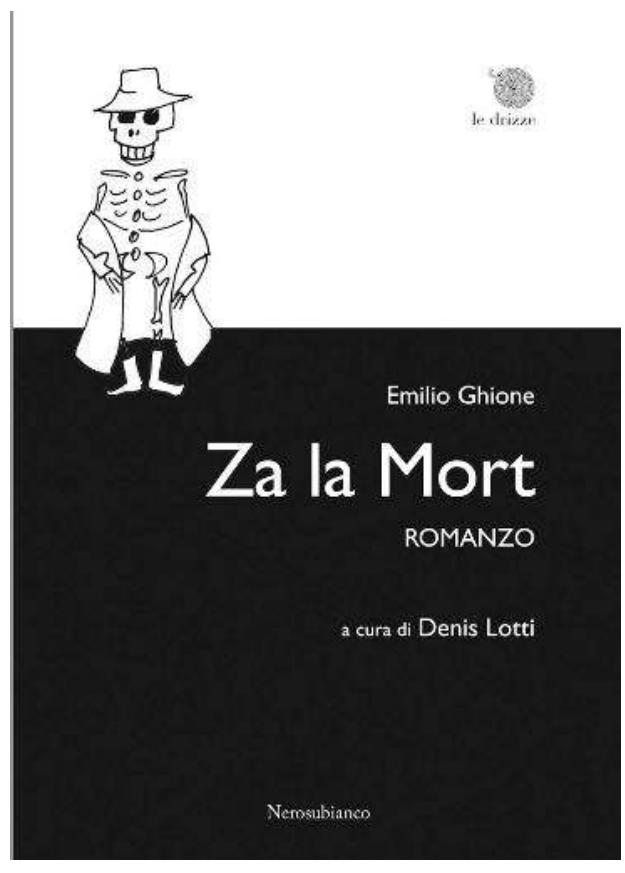

2 Parigi, "fra quel grigiore 1925" (15). Un giovane visconte, criminalmente privato dell'eredità che gli spettava, diventa apache, ma pur sempre restando nell'intimo il perfetto gentiluomo ch'era, guardando costantemente gli altri "dall'alto della propria rettitudine" (29). Conosciuto con il nomignolo di Za la Mort - che ci vien detto significare Viva la Morte - non tarderà ad innamorarsi d'una donzella dalla "faccia rovente di luce" (19), suo perfetto pendant, ribattezzata logicamente $\mathrm{Za}$ la Vie. L'amore, anche tra apache, essendo per definizione impossibile presso gli eroi romanzeschi, non mancherà d'intervenire il destino, sotto le spoglie di tale Perla Cristal, crogiuolo di tutti i vizi e tutte le crudeltà, per trasformare Za la Mort in forzato e Za la Vie in cretina, idiota, imbecille, nel senso più tecnico possibile di privata della ragione. Il tutto su sfondo di complotti e crimini scioccanti ed inconcepibili orditi da una tentacolare società segreta diretta da un misterioso quanto intelligentissimo paralitico, di cui Perla Cristal è una degli agenti. La storia, con le variazioni necessarie, segue il canovaccio dumasiano standard provvisto dalle disgrazie del conte di Montecristo. Il forzato ritornerà, non dopo aver trovato un amico e sostegno nell'"avvocato", altro ergastolano che, novello abate Faria, gli spiega il crimine del quale è stato vittima. L'eroe "comico, furbesco, incorreggibile, caustico" (80), si trasforma in principe indiano (notasi, di nome Gandhy), pronto a stupire la società parigina con quel "credito illimitato" (133) di cui dispone, esattamente come tale Dantès prima di lui, ma per aver trovato un fiume d'oro nelle profondità d'un isola, tipo del Diavolo, dove la giustizia (relativamente parlando) l'aveva spedito a morire in miniera. Grazie a tale ricchezza favolosa, l'eroe minaccia alle nazioni "la bancarotta del Globo" (108) per inondazione d'oro e, scambiate verghe innumerevoli di metallo giallo con dollari e sterline, si compra una bella vendetta mentre alla ricerca dell'amor suo, sperduta e smemorata nei meandri della metropoli.

3 Nella lotta contro i “purpurei gavazzatori” (81), responsabili delle sue disgrazie - tra i quali spicca un Cinese in kimono di nome Siky-Ho, simbolo di fatalità, che parla mongolo e dopo qualche capitolo si trasformerà in giapponese - lo "scheletro parlante" (97) che è il nostro eroe si aggira in una Parigi fiabesca, mentre la narrazione, in un 
delirio di esterofilia, farcisce ogni frase di termini francesi tutti rigorosamente e meravigliosamente storpiati, quasi senza eccezione alcuna (cigalle, magazain, l'Ambigû, bloch-notes, coch-tail, clachson, flich, rhumm, la torre Eifell, e mille altri...). Za la Mort, obbligatoriamente, piange come il Corsaro Nero, davanti alla sua amata rimbecillita, si rivela, come Montecristo, smettendo le vesti di Indiano favolosamente ricco per tornare apache davanti a Perla Cristal, e poi si vendica tramite terzi, ancora come Montecristo, lasciando Perla Cristal cadere nelle mani del geloso Siky-Ho come Caderousse in quelle di Benedetto. E così sia. Ma come sempre in tal tipo di narrazione, l'interesse non sta in qualche presunta come improbabile originalità dell'intreccio ("L'intreccio d'una film avventurosa" (28), per dirla al femminile come l'autore) quanto in altri luoghi meno ovvi. O forse, anche troppo ovvi in questo caso. La qualità principale del romanzo risiede in uno stupefacente accavallarsi di aggettivi e d'avverbi da far rivoltare Hemingway nella sua tomba, di descrizioni roboanti, di esageratissime, verbosissime, complessissime locuzioni annegate negli incisi, dove dietro ogni epiteto démodé pare sentire un gran suonar di grancasse e rullar di tamburi. Metafora della narrazione, il processo dell'eroe: "Il Presidente avvisò l'accusato di essere più conciso. Non avesse mai avuta una simile, peregrina, trovata" (41). Non si scrive più così. Magari è un'ottima cosa. Ma rimmergerversi talvolta non è un esercizio privo di piacere o di profitto, e non si può, in questo, non schierarsi decisamente contro l'autore della postfazione, che deride "la narrativa scadente di Ghione, almeno sotto il profilo letterario" (232). Eccessi di questo genere sfuggono per definizione ad ogni giudizio prettamente tecnico.

Dettaglio interessante, delle tracce di critica sociale fanno qua e là capolino, o quando l'autore, novello Albert Londres, compiange "coloro che furono, che la Società malvagia e vile li uccide senza dar loro la morte" (97), o quando denuncia "la valuta, la moneta, il perchè del lavoro, della vita", come "il pilastro demoniaco che regge le sorti umane" (118). E che dire di certe domande retoriche alle quali si cerca ancora invano risposta: "Società, quando, invece di punire, guarirai? Quando al posto dei carcerieri si vedranno infermieri? Quando i medici sostituiranno i giudici?" (137) Viva i bei sentimenti, che sopravvivono ancora solamente nelle riedizioni dei vecchi romanzi popolari.

5 Maggior cura potrebbe essere stata data alla rilettura del testo. Numerosi refusi distraggono e talvolta innervosiscono. Qualche frase monca di troppo, soprattutto verso la fine, guasta l'innocente piacere della lettura. Malgrado ciò non si può che raccomandare la riscoperta di questo delirante esempio di appendicistica nostrana, o piuttosto, di novellizzazione avant la lettre, visto che si tratta di un romanzo, come ce l'insegna l'utile postfazione di Dennis Lotti, piena di informazioni curiose, scritto dal regista ed attore stesso che aveva portato allo schermo le avventure dell'eroe eponimo ai tempi d'oro del cinema muto. Che il romanzo popolare italiano debba molto, se non tutto, a quello dell'ottocento francese, è cosa già nota. Che abbia potuto produrre tali sorprendenti e gradevoli variazioni sul tema, in compenso, merita d'essere ricordato. 


\section{AUTORI}

VITTORIO FRIGERIO

Dalhousie University 